\title{
Scientifically proving God's existence
}

John Taylor ${ }^{1}$,

\begin{abstract}
:
It is possible to scientifically test/prove the existence of an ultimate form of consciousness. However, it is not possible to scientifically conclude whether this supreme form of consciousness, is indeed God (which is defined as the creator and ruler of the universe). Moreover any contention, which seeks to prove the existence of God scientifically, must do so by modifying arguments which have premises that when added together undoubtedly lead to the conclusion that God exists. The premises themselves must be modified, so that they can be tested by the scientific method. It is also implied at the end of this paper, that the methods suggested in this article, could be applied to other areas of philosophy in order to empirically test various ideas.
\end{abstract}

Keywords: God; Quantum Mechanics; Wigner's friend; Scientific Method

${ }^{1}$ AFFILIATION: None, EMAIL: johnjoetaylor78@gmail.com , ORCID: 000-0003-4592-2589 
TABLE OF CONTENTS

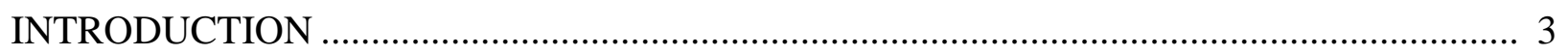

SECTIONS:

1. WIGNER'S FRIEND AND IT'S SCIENTIFIC CRITERIA ...................................... 5

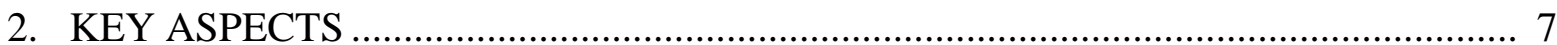

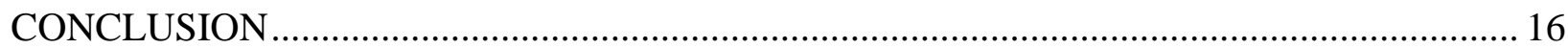

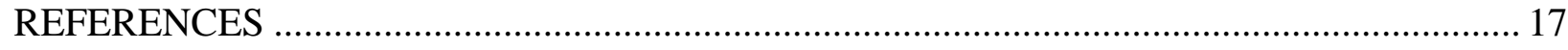




\section{Introduction:}

The issue, of substantiating, the existence, God has been a major problem, in religious apologetics. Philosophers of religion, have attempted, to erase any doubt that God exists, by trying show that the existence of God is logically necessary. This has been attempted by formulating so called 'ontological' arguments. Ontological arguments, generally insist that since God is the "greatest being to be conceived", he has to be real since by existing only in the mind, he would be less great than a being which existed in reality ${ }^{2}$. Despite, being an interesting attempt at proving God's existence, the majority of philosophers regard the argument, as a failure, for a variety of reasons, but largely because existence cannot be regarded as a predicate. Another, problem, with this argument, is that the premises, in which it is predicated upon are not absolutely true, and thus the conclusion that God exists does not follow. For example the predicate, that it is greater to exist, in reality than in the mind, is arguably a subjective opinion. Hence, overall, it seems rather improbable, that a successful argument, could be made that God exists, due to his definition being a logical necessity.

Although all of the current formulations of the ontological argument, ultimately fail, in providing, an absolute proof, of God's existence, perhaps a more reasonable approach to proving God's existence, with a very high level of certainty, is to formulate a proof that is scientific. This, type of approach is much more beneficial, than trying to authenticate God's existence, by showing that God, is a logical necessity, since a scientific validation, doesn't need to be an absolute proof. Rather, an empirical confirmation must know with a tremendously high level of assurance that, something is the case ${ }^{3}$. Inspired, from this line of thinking this paper aims to, outline, a possible method by which a scientific authentication of God's existence could be reached. A conclusion, will be made, as to whether, this could in principle confirm God's existence empirically, as well as exploring the implications surrounding the conclusion reached, and various other ideas.

Now your immediate reaction, to such a proposal might be to think that, the suggestion, is being made that in order to scientifically prove God's existence, experimenters should detect him, like detecting a particle in a laboratory. Don't' worry, this idea is inherently, foolish. By nature, God cannot be open to the direct observation of science because his immaterial essence, prevents examination by a system, which strictly deals with natural occurrences. In order, to get around this issue, a scientific proof of God's existence, should not aim to directly (scientifically) observe him, but to instead, use a series, of premises, that are in themselves scientifically testable, and will if empirically proven, and added together, lead to the conclusion that God exists. Essentially, the argument would have to be formulated in such a way that if the premises are true the conclusion undoubtedly follows. Hence, a scientific proof of God's existence would be reached, since an argument would have been constructed in which, the premises are scientifically justifiable and if true would unquestionably lead to the existence of God. Moreover an important, distinction must also be made, between what is being proposed here, and the premises, used for inductive arguments (i.e. design argument). Inductive arguments

\footnotetext{
2 Plato.stanford.edu. (2019). Ontological Arguments (Stanford Encyclopedia of Philosophy). [online] Available at: https://plato.stanford.edu/entries/ontological-arguments/ [Accessed 16 Aug. 2019].

${ }^{3}$ Plato.stanford.edu. (2019). Scientific Method (Stanford Encyclopedia of Philosophy). [online] Available at: https://plato.stanford.edu/entries/scientific-method/\#LogMetCriRes [Accessed 16 Aug. 2019].
} 
similarly use premises made from observation, with the only difference being that these premises can't be scientifically justified ${ }^{4}$.

Since this article, is endeavouring, to demonstrate that God's existence can be proven empirically it is necessary to define what is meant by "God". For the purposes of this article, God shall be defined as the creator, and ruler of the universe. This definition shall be used, since it is a description that is typically found in all of the major monotheistic religions ${ }^{5}$.

With all of this in mind what is needed, is an ideal scientific field, to meet these demands. It turns, out a perfect area, to meet these requirements, is quantum mechanics and in particular Wigner's friend. Wigner's friend is a thought experiment, created by Nobel laureate, Eugene Wigner, and is sometimes used, to argue, for the existence, of God. Upon analysis of this thought experiment, it becomes apparent that it is composed of scientifically testable premises. To, see why this is the case, it is necessary to unpack Wigner's friend, and to specify, in detail, the scientific criteria, it uses. As will be done in the next section.

Moreover, in order for a premise, to be considered scientifically testable, it needs to be capable of being tested, by the scientific method, which can involve formulating a hypothesis and probing it by an experiment ${ }^{6}$. Moreover, some key underpinnings of the scientific method involve:

1) A hypothesis, being testable and falsifiable

2) An experiment, should include a dependent and independent variable

3) An experiment, should include an experimental group and a control group. ${ }^{7}$

Obviously, now there are exceptions to these specifications, as to what makes an experiment scientific. If an experiment, designed, to test premises used to build a scientific proof of God's existence, turns out to be one of these exceptions, this will be alerted, and explained why in this case it is still scientific. An example, of one of these exceptions, is the search for dark matter. A substance which could theoretically be observed. However if it is not it found, then it cannot be stated that it doesn't exist. Therefore, the search for dark matter is not falsifiable, and does not fully meet the requirements of the first point, but is nevertheless still considered to be scientific.

It must also, be said that this paper, will not aim to ensure that the scientific experiments, and methods are practical, but rather are the types of experiments that could be conducted in principle, in order to demonstrate that it is feasible to prove the existence of God scientifically.

\footnotetext{
${ }^{4}$ En.wikipedia.org. (2019). Existence of God. [online] Available at: https://en.wikipedia.org/wiki/Existence_of_God\#cite_ref-46 [Accessed 22 Aug. 2019].

5 Lexico Dictionaries | English. (2019). God | Definition of God in English by Lexico Dictionaries. [online] Available at: https://www.lexico.com/en/definition/god [Accessed 22 Aug. 2019].

6 Plato.stanford.edu. (2019). Scientific Method (Stanford Encyclopedia of Philosophy). [online] Available at: https://plato.stanford.edu/entries/scientific-method/\#LogMetCriRes [Accessed 16 Aug. 2019].

7 Bradford, A. (2019). What Is Science?. [online] livescience.com. Available at: https://www.livescience.com/20896-science-scientific-method.html [Accessed 17 Aug. 2019].
} 


\section{Wigner's Friend and its Scientific Criteria:}

With the advent of quantum mechanics, in the early $20^{\text {th }}$ century, there was a paradigm shift in the assumptions that were held about the world. A concept, which perhaps smashed our presuppositions of nature the most, is that of quantum superposition. This principle claims that a quantum system (e.g. a particle), has the ability to be in multiple states at the same time, prior to being measured ${ }^{8}$. Exactly, how this should be interpreted, has not yet been solidified ${ }^{9}$.

Some physicists found this idea hard to believe. In 1935 Erwin Schrodinger, one of the founders of quantum mechanics, devised a thought experiment, to drive home the strange rules of his theory ${ }^{10}$. His thought experiment imagines a cat being placed, inside a box with a vile of poison, along with a Geiger counter, and tiny piece of radioactive uranium. If the radioactive substance, decays and is detected by the Geiger counter, the flask of poison is smashed, by a hammer (via a relay system), which in turn kills the cat. Now, since the superposition principle, dictates that a quantum system can be in multiple states simultaneously (when not being measured) and because this principle applies to the phenomenon of radioactive decay, it should be considered as having both decayed and not decayed at the same time (if left unobserved). This superposition state, therefore translates to the cat being both dead and alive, in the moment that the box is left unopened, since its fate as explained earlier, is linked to whether the substance decays or not ${ }^{11}$.

An extension of Schrodinger's cat was formulated by Eugene Wigner, called "Wigner's friend". Wigner's friend, posits, a colleague of Wigner's, working in a separate laboratory from his own. He has been given the task of performing the Schrodinger's cat thought experiment and recording the outcome. To the person, outside of the lab, his friend's measurement is a unitary interaction, which essentially means that the recorded state, and the state that the cat is in, are effectively the same piece of information. Wigner, himself, can observe the experiment, though he is provided with no information about his friend's measurement or the recorded outcome. Without this information and with no means of interacting with the particle, quantum mechanics forces him to assume that the fate of the cat is in a superposition of being both alive and dead at the same time. When Wigner asks his friend for the results of the experiment, from his perspective the superposition state of the cat now collapses to a single state of being either dead or alive. Wigner, and his friends record, now share, the same superposition state as that of his friends measurement.

\footnotetext{
8 Dirac, P. (1947). The Principles of Quantum Mechanics (2nd edition). Clarendon Press. p. 12.

${ }^{9}$ chlosshauer, M., Kofler, J. and Zeilinger, A. (2013). A snapshot of foundational attitudes toward quantum mechanics. Studies in History and Philosophy of Science Part B: Studies in History and
}

Philosophy of Modern Physics, 44(3), p.230.

10 Schr dinger, E. (1935). Die gegenw rtige Situation in der Quantenmechanik. Die Naturwissenschaften, 23(48), pp.807-812.

11 Schr dinger, E. (1935). Die gegenw rtige Situation in der Quantenmechanik. Die Naturwissenschaften, 23(48), pp.807-812. 
This creates a curious implication, during the time in which Wigner is forced to view the cat as being in a superposition, his friend views the same cat as having a defined state. But since both points of view must be regarded as equally valid, this is where an apparent paradox comes in. When exactly did the collapse of the cat's superposition occur? Was it when his friend had finished his measurement or was it when the information entered into Wigner's consciousness? ${ }^{12}$ Some have claimed that the only way to solve this paradox is to invoke an ultimate observer ${ }^{13}$. An ultimate observer, is essentially a consciousness, that is not associated with the experiment and has the ability to perceive the experiment from the outside, which is arguably God. By being outside, or separate, it is not part of what composes the paradox, since it is detached from the physical system. And therefore, there is no paradox as to when it collapses, because there is now a defined place as to when it will collapse, since something from outside the whole experiment has been introduced.

When looked, at in detail, it becomes apparent that this thought experiment, is constructed out of scientific premises, which if proven would undoubtedly lead to the conclusion that God exists. The premises shall be listed and an explanation given as to how, when these premises are added together, they unquestionably arrive at the conclusion that God exists:

The first premise, that needs to be substantiated, is whether 1) metaphysical consciousness is responsible for wave function collapse. This needs to be confirmed since by knowing that immaterial consciousness in general causes collapse, it can be used later as a spring board to infer that a higher consciousness, i.e. God, is responsible for there not being a paradox that should have been there, since if an immaterial consciousness is needed to collapse the wave function in general it must be needed to collapse the wave function in Wigner's friend, which is understood to be in a paradox in relation to when it collapses. This therefore allows for the creation of premise 2. The second premise, which is imperative to justify is that there should be a paradox (as defined in Wigner's friend) and that the only possible way in which there couldn't be one would be by invoking an ultimate observer (God). This is imperative to verify, because by knowing that there should be a paradox, and that this can only be solved by invoking God as an ultimate observer, then if no paradox is found we know that God is real, because the only way in which the paradox couldn't be there, would be by invoking an ultimate observer (God).

Moreover it needs to be shown, that the paradox cannot just remain, since this would mean that there is no need to invoke God to solve it, because it can just remain in a paradoxical state. This contradictory state can come in two forms, 1) separate realities coexisting, and 2 ) it can exist in one reality as a paradox, due to it being a philosophical contradiction. The final premise that needs to be substantiated, is that the wave function of the initial particle in Wigner's friend (and the first observer) has

12 Informationphilosopher.com. (2019). [online] Available at:

http://www.informationphilosopher.com/solutions/scientists/wigner/Wigner_Remarks.pdf [Accessed 22 Aug. 2019].

13 Informationphilosopher.com. (2019). [online] Available at: http://www.informationphilosopher.com/solutions/scientists/wigner/Wigner_Remarks.pdf [Accessed 22 Aug. 2019]. 
undoubtedly collapsed. Additionally this should be deduced from the perspective of observer 2. This would ultimately confirm that God exists, since if it is found that the wave function of the particle has definitely collapsed, down to one state (from all perspectives), this would mean that there is no paradox (as defined in Wigner's friend), and would therefore mean that the conclusion has to be reached that God exists, since as would have been confirmed in premise 2 , the only possible way in which there couldn't be a paradox would be the invocation of God as an ultimate observer. If all of this were done scientifically, then an empirical proof of God's existence would be formulated. The next section will show that theses premises can be scientifically authenticated.

\section{Key aspects:}

1) Metaphysical consciousness causes collapse:

The first premise that needs verifying is whether consciousness causes collapse. It is necessary to define consciousness as a metaphysical awareness. By knowing that a metaphysical cognizance can induce wave function collapse, this will allow us to infer that a higher form of consciousness, in the form of an ultimate observer (otherwise known as God), is responsible for reconciling the paradox of when exactly collapse occurs in the Wigner's friend thought experiment. In the manor that was specified previously.

In order to determine that metaphysical consciousness triggers wave function collapse it is important, to be certain that other definitions of consciousness, which are not metaphysical, don't have a causal link to the phenomena of wave function collapse. One such definition, is Integrated Information Theory (IIT). This idea, postulates, that, consciousness is not binary, but rather, operates on a scale. Ultimately this indicates a form of pansychism ${ }^{14}$. Additionally, IIT sets itself apart from various immaterial theories of consciousness, by claiming to be able to precisely define how consciousness, can be measured. According to IIT, a system is sentient, if it possesses an attribute known as, $\Phi$, or 'phi' ${ }^{15}$. The theory states, that systems, with high 'phi' (e.g. the human brain), are highly conscious, and in turn have highly complex and meaningful experiences. In contrast, systems, with low phi, have a small amount of consciousness, and thus, only experience the world in simple and rudimentary terms. Finally systems, with zero 'phi', have no consciousness ${ }^{16}$.

\footnotetext{
14 Philosophynow.org. (2019). The Integrated Information Theory of Consciousness | Issue 121| Philosophy Now. [online] Available at: https://philosophynow.org/issues/121/The_Integrated_Information_Theory_of_Consciousness [Accessed 18 Aug. 2019].

15 Philosophynow.org. (2019). The Integrated Information Theory of Consciousness | Issue 121| Philosophy Now. [online] Available at: https://philosophynow.org/issues/121/The_Integrated_Information_Theory_of_Consciousness [Accessed 18 Aug. 2019].

16 Philosophynow.org. (2019). The Integrated Information Theory of Consciousness | Issue 121| Philosophy Now. [online] Available at: https://philosophynow.org/issues/121/The_Integrated_Information_Theory_of_Consciousness [Accessed 18 Aug. 2019].
} 
Phi, is based on information, integration, and maximality. According to IIT in order for a system, to have a high phi, this depends partly on how much of the information it has is about itself ${ }^{17}$. The more information a system, has about itself the higher its 'phi', and vice versa. Furthermore, this information must be based on the system's own causal powers, and not on symbolic conventions. Moreover, for a system to have a high level of consciousness a high level of 'integration' is required. This means that the information contained within a system, must be highly dependant, on the interconnections between the parts of that system. Lastly maximality is needed. This means that in order for a system to be highly conscious, it must have more integrated information than any overlapping system, including its own parts ${ }^{18}$. Clearly, according to IIT consciousness is something that is measurable.

Now since IIT states that some objects, are more conscious than others, and that consciousness can be defined and measured by a precise mathematical quantity, this allows for the possibility of actually creating a conscious object. Based on this principle, a variation of the double slit experiment, involving Nano computers has been proposed. In this experiment a test group of Nano computers(with high phi) are sent through the slits, followed by a control group being sent through with low phi, meaning that there is little or no consciousness. If consciousness as specified by IIT causes collapse, it would be expected for there to only be an interference pattern, with the control group, which has little or no phi. This therefore shows that in principle the hypothesis that consciousness causes collapse, as defined by IIT, is scientifically testable.

Although, this experiment, succeeds in developing a mechanism, for testing the hypothesis that consciousness (as defined by IIT), causes wave function collapse, the way it proposes to do so, is too incommodious. However, a more viable option would be to adapt a recently published experiment, which makes the proposal of placing a microorganism in superposition, by virtue of positioning it on top of an electromechanical oscillator, in order to test the hypothesis in question ${ }^{19}$. The authors of the paper indicate that the ideal procedure to place a microorganism, in a state of superposition, is to use the motion of a mechanical oscillator to superimpose a ground state and an excited state, of that organism ${ }^{20}$. The authors, further posited that the motion of a microorganism, can be entangled with an internal state of an electron inside of $i^{21}{ }^{21}$. This research suggests that it is an accomplishable milestone, to place a living organism in superposition.

\footnotetext{
17 Philosophynow.org. (2019). The Integrated Information Theory of Consciousness | Issue $121 \mid$ Philosophy Now. [online] Available at: https://philosophynow.org/issues/121/The_Integrated_Information_Theory_of_Consciousness [Accessed 18 Aug. 2019].

18 Philosophynow.org. (2019). The Integrated Information Theory of Consciousness | Issue 121| Philosophy Now. [online] Available at: https://philosophynow.org/issues/121/The_Integrated_Information_Theory_of_Consciousness [Accessed 18 Aug. 2019].

${ }^{19} \mathrm{Li}, \mathrm{T}$. and Yin, Z. (2016). Quantum superposition, entanglement, and state teleportation of a microorganism on an electromechanical oscillator. Science Bulletin, 61(2), pp.163-171 ${ }^{20} \mathrm{Li}, \mathrm{T}$. and Yin, Z. (2016). Quantum superposition, entanglement, and state teleportation of a microorganism on an electromechanical oscillator. Science Bulletin, 61(2), pp.163-171.
}

21 Physics World. (2019). Could 'Schrödinger's bacterium' be placed in a quantum superposition? Physics World. [online] Available at: https://physicsworld.com/a/could-schrdingers-bacterium-beplaced-in-a-quantum-superposition/ . [Accessed 22 Aug. 2019]. 
Now, since research has shown that Nano computers could potentially be built to the size of a virus ${ }^{22}$, logically it follows that one could place a Nano computer (of that size), or of a slightly larger magnitude, on top of an oscillator that is capable of putting it into superposition. This goal is much more attainable than that of designing Nano computers, at the size required to generate an interference pattern, in a differentiation of the double slit experiment. Furthermore rather than endeavouring to place the Nano computers into a superposition of its ground state, and an excited state, it should be placed into a superposition of a low energy state, and an excited state, in order to allow the computer to function.

Some of the Nano computers, will be programmed with high $\Phi$ (the test group), whereas some with no, $\Phi$ (the control group). As in the experiment involving the entanglement, of the motion of a microorganism, with an electron inside of it, similarly, one could artificially create, an electron, which is stored internally, inside the Nano computer to be entangled with its motion.

This set up therefore allows for the hypothesis that consciousness, as defined by IIT induces collapse to be tested, because its entanglement is dependant upon the superposition of the Nano computer, being in an excited state or low energy state, which would arguably not occur, in the test group which contains Nano computers with a high $\Phi$. This is because according to IIT it should have collapsed to one state (whereas for no $\Phi$, entanglement would occur). Therefore, if one is unable to entangle, an internal electron with the motion of a Nano computer, with high $\Phi$, but they are for low $\Phi$, one would have to reach the conclusion that this was due to the Nano computer collapsing to a particular state (either low energy or high energy), and that this must be due to it being 'conscious', as defined by IIT, since this did not occur in the Nano computer with low $\Phi$.

Although this experiment would be able to rule out whether, an IIT type consciousness causes wave function collapse, it is severely limited. This is because; it can't establish whether a metaphysical form of consciousness causes collapse. However, it is possible to verify for certain that an immaterial consciousness generates collapse, and in turn rule out all other definitions of consciousness for causing collapse, by performing an experiment on the brain.

The experimental scenario would be based around a volunteer, having a superposition of multiple different images sent to the subject's brain. Throughout the duration of the experiment, the signals are being monitored by a scientist, to ensure a state of superposition, is maintained. It must be said that monitoring the signals, does not involve making a direct observation of them, but rather looking for circumstantial effects that would indicate whether there is a condition of superposition. For instance, interference, which could potentially be observed by placing a microscopic pair of slits within the brain, to be used to detect superposition, by monitoring whether an interference pattern, is created on a microscopic detector screen further back, as the signals come in to the brain. During this experiment the decoherence effect will be prevented. This could possibly be achieved by modifying the brain. For instance, one could imagine, in the future, with the development, of trans humanist, technology, that the brain could be modified in such a capacity, that human consciousness is still there, whilst, decoherence, of the incoming brain signal to an Eigen state (perhaps exclusively), is prevented. It is also, worth noting that the brain signals, may only have to be placed in a superstition state for a very short time span. Ultimately the duration of the superposition state of the

22 The UCSB Current. (2019). A Tiny Machine. [online] Available at: https://www.news.ucsb.edu/2016/017349/tiny-machine [Accessed 22 Aug. 2019]. 
signals, just needs to be long enough, for us to know that a metaphysical object has provoked collapse.

Now if the wave function were to collapse down to one particular image (which is being monitored by an experimenter) when the signals enter into the volunteer's brain, the only reasonable conclusion that one could reach is that this occurred due to an observation made by a metaphysical mind. This is because by ruling out the possibility of collapse occurring due to decoherence with the matter of the brain (by some how preventing it), the only other explanation, for the wave function collapsing to one particular state would be observation made by a metaphysical mind, since the other alternatives (which all involve matter), couldn't possibly be the cause (since they were prevented, from causing collapse, by stopping the decoherence effect). Essentially, by preventing decoherence, all explanations, of collapse, relating to interaction by matter, would be ruled out, if the incoming signals were to collapse anyway.

Moreover the control group that should be used; is to compare it against non-conscious entities, such as animals or robots, or even something with a low IIT. This experiment, ultimately would cut out all other definitions of consciousness, as existing, and as the cause of wave function collapse, because, by stopping decoherence, the experiment has determined that collapse by interaction with matter cannot be the correct explanation, and in turn verified a metaphysical explanation, which means that the others can't be correct since one has been verified.

In closing, the experiments outlined in this section have shown that this premise is empirically testable (and thus contributes to scientifically testing the hypothesis of God). Although, the last experiment mentioned, is not falsifiable, and the independent variable (metaphysical consciousness) used in this experiment, cannot be known to exist prior to the experiment, it is still scientific, partly due to there still being a test group and a control group. Moreover the experiment is still scientific since it is confirmable in the same capacity that the search for dark matter is, which is also not falsifiable in the same sense as the experiment mentioned here (This was mentioned in the introduction as an exception to the criteria for scientifically testable hypothesis, but is still nevertheless scientific). Therefore, although the experiment in question doesn't perfectly meet the criteria outlined in the introduction to render it scientific, it is nevertheless an empirically testable hypothesis. Primarily, because the areas in which it doesn't flawlessly meet the requirements, are valid exceptions.

Additionally, if this experiment, were to confirm, that metaphysical consciousness does indeed effectuate wave function collapse, then a key milestone has been accomplished in empirically substantiating whether God exists. This scientifically ratified premise, can then be used in the fashion explained, earlier to scientifically deduce that God exists with the empirical verification of other required premises, thus contributing to making the hypothesis of God scientifically testable. It is also worth noting, that this experiment, could potentially be utilized, to determine, whether, any being or thing is conscious, by being modified, to test whether a metaphysical awareness causes collapse in these objects. Therefore, this experiment, not only has value in providing a method to scientifically test the first premise of this argument (which depending on the result could potentially be used to scientifically prove the existence of God), but is also potentially beneficial in other spheres of scientific research. 
2) The Wigner's friend thought experiment, suggests that there should be a paradox there (as is specified in Wigner's friend), and that the only possible way in which there couldn't be a paradox, is the invocation of an ultimate observer (God):

As was specified earlier, it is necessary to scientifically determine, that there should be a paradox present (as defined in Wigner's friend), and that the only way in which there couldn't be a paradox is by the invocation of a type of ultimate observer. In summary, this is needed because, if one were to reach the conclusion that there should undoubtedly be a paradox, and the only solution to it is God, and then it is found that there is no paradox, since it has been determined that the wave function of the particle in Wigner's friend has definitely collapsed (This means that there can't be a paradox since there can be no paradox as to when it collapses in the chain of observers in Wigner's friend, if it has been proven that there is no doubt the decayed particle has collapsed to one state), then the only conclusion that could be reached is that God is responsible, and in extension that God exists.

It turns out that this postulate is scientifically confirmable. Primarily, because, any contention, which proposes that there isn't a paradox, would have to argue this by condemning premises, which can in principle be scientifically upheld. This is because, the paradox itself comes into fruition due to it being composed of contradictory scientific/natural premises, and thus the idea that there isn't a paradox can be tested scientifically. This is because the only way one could make a sincere claim that there is no paradox is denying empirically certified premises, since these premises are what compose the paradox in the first place.

The first premise that is typically attacked in an effort to disprove that there is a paradox is the assumption that consciousness causes collapse. Additionally, a case is often made that there is no paradox since, the premise, that the original observer, is in a state of superposition in the first place, is false. In relation to the first premise which is ordinarily condemned; claims have been made that consciousness doesn't generate collapse, because conscious observation does not involve interaction and is thus not a measurement and hence, no paradox is created. As well as the claim that some other mechanism, other than consciousness is responsible for the phenomenon of wave function collapse ${ }^{23}$. A further argument in relation to the first premise which is typically castigated would be to, attempt to debunk the notion that a paradox is there, by claiming that, observation becomes personalised, and thus no paradox is there, just missing information ${ }^{24}$. In relation, to the second premise, that is typically attacked, one could claim that no paradox is present, because the wave function unambiguously collapses, when observed by the first observer, in the box with the cat. Self evidently these arguments, are attempting to disrupt premises, that can be proven empirically, one way or the other, and hence the argument, that there is no paradox, is scientifically testable because the premises being attacked are scientific in nature. For instance any argument, that consciousness doesn't cause collapse, would have been disproven with the brain superposition signals experiment that was mentioned earlier. The same also, goes for arguments, which attempt to disprove a paradox being present, by suggesting that the

23 YouTube. (2019). Wigner's Friend Paradox: Is Observation Inherently Flawed?. [online] Available at: https://www.youtube.com/watch?v=5AodzEpvzZw [Accessed 22 Aug. 2019].

24 YouTube. (2019). Wigner's Friend Paradox: Is Observation Inherently Flawed?. [online] Available at: https://www.youtube.com/watch?v=5AodzEpvzZw [Accessed 22 Aug. 2019]. 
superposition collapses when observed by the first observer. It turns, out that arguments, that use this line of reasoning are also scientifically testable, since such a premise, can be confirmed by an experiment, that will be mentioned later on in this paper.

Another premise, that could be attacked, is that the Copenhagen interpretation is correct, and instead another theory, which rules out observation all together for being responsible for collapse, is accurate. However, even this premise, could be upheld scientifically, by virtue of the results of the experiment, involving a superposition of signals being sent to the brain. Such an experiment would show that all interpretations which by framework claim that it is impossible for consciousness (under all definitions) to cause collapse to be wrong. If the results of the experiment, conclude that an immaterial consciousness does indeed cause wave function collapse. An example of a theory, which by framework doesn't support consciousness-causing collapse, is the Everettian formalism, of the Many-worlds interpretation and the De-broigle-Bohm theory ${ }^{2526}$. Moreover, the experiment would firmly falsify these interpretations, since by verifying that metaphysical consciousness causes collapse, it means that these theories cannot be correct, since by nature these interpretations don't allow for non-physical consciousness to cause collapse, and thus if it has been confirmed that immaterial consciousness does induce collapse then these interpretations cannot be valid because essential to these theories' description of quantum mechanics, is the impossibility of metaphysical consciousness causing collapse. And a system which holds as an essential necessity in order for it to function (meaning that the system would be a totally different and distinguishable theory, if that aspect were no longer present), the proposition that consciousness (under all definitions), is not responsible for collapse when the experiment in question has verified that it is responsible, cannot be correct, unless the system itself is modified. Hence when viewed holistically, these alternative interpretations cannot be correct. Therefore, the experiment that is being discussed here, would discredit numerous interpretations that have frameworks, that deny consciousness (in any form), can trigger wave function collapse, which includes the Many-worlds interpretation and Bohmian mechanics. This thus shows that even an argument against this premise based on the idea that another, interpretation of quantum mechanics is correct, cannot evade scientific testability.

Furthermore, by confirming, that collapse, is due to an immaterial object, one has effectively eradicated interpretations that might in principle adopt IIT as its cause of collapse. Since, we know that collapse occurs due to metaphysical consciousness, hence an argument, cant be made that there is no paradox, because a certain interpretation might be correct, which adopts a different form of consciousness (e.g. IIT), as responsible for causing collapse, because this experiment has confirmed that collapse occurs due to metaphysical consciousness. Additionally, even if various theories, that have frameworks that deny that consciousness induces collapse, are modified to incorporate the verified feature that immaterial consciousness effectuates collapse, the paradox still stands, since any theory, that postulates a metaphysical consciousness causing collapse, would allow for that paradox to work.

An additional, necessary factor, to confirm, is that the only solution to the paradox in Wigner's friend, is the invocation of an ultimate observer (God). Again, it turns out that this is empirically verifiable. The invocation of an ultimate observer, would mean that no paradox is present, since its observation of the whole system, from its privileged position,

25 Plato.stanford.edu. (2002). Many-Worlds Interpretation of Quantum Mechanics(Stanford Encyclopedia of Philosophy). [online] Available at: plato.stanford.edu/entries/qm-manyworlds/ [Accessed 16 Aug. 2019].

26 Plato.stanford.edu. (2001). Bohmian Mechanics (Stanford Encyclopedia of Philosophy). [online] Available at: plato.stanford.edu/entries/qm-bohm/ [Accessed 16 Aug. 2019]. 
would allow for unambiguous collapse, since its select position, would give it a platform, for it to observe it from an outside perspective, which would mean that there would be no contradiction in perspectives. Moreover, this can be tested empirically by scientifically ruling out all of the other explanations, for how the paradox could be reconciled until we know that God is responsible for it. Any explanation, which is not an ultimate observer, and is instead scientific, by definition would be a naturalistic explanation (which allows for them to be tested scientifically, as will be explained later). Therefore, to try to reconcile the paradox, without the use of an ultimate observer would have to attack the naturalistic premises used to argue that Wigner's friend proves the existence of God, by virtue of being a naturalistic explanation. For instance, one could make the claim, that there is no need for an ultimate observer, and it just stops at the first observer. This claim would have to deny the predicate that there is a paradox, which as was shown earlier, is scientifically verifiable. Clearly, any other attempt to show that God doesn't, cause collapse, would have to resort to attacking the relevant premises used to build up this argument. However as was established previously these premises, are scientifically provable, and thus by ruling out the objections one by one, we would reach the conclusion that God, or an ultimate form of observer, is the only solution to the paradox.

Therefore, it is clear that it can be scientifically tested and proven that there should be a paradox, and that the only explanation for not finding a paradox there, would be the invocation of an ultimate observer. All of this is invaluable in scientifically proving the existence of God, as it was described previously.

\section{3) The paradox cannot just remain as a paradox:}

Another integral area that needs to be scientifically confirmable is whether the paradox (as defined in Wigner's friend) can simply remain as a paradox. This means that the contradicting realities, of exactly when the particle collapses, in Wigner's friend can just coexist. It is crucial that this premise can be scientifically tested, since it would confirm that the problem of having a paradox couldn't be solved by stating that this paradoxical state is the way that reality is. As was mentioned, earlier the paradox could potentially remain, by either: 1) the separate realities of when it was measured just coexisting, or 2) the whole paradox just existing as a philosophical contradiction.

It turns out that an experiment, conducted in 2019 , was purportedly able to scientifically test the hypothesis in question (in the sense that the separate realities could just coexist). The experiment concluded that the paradox could just remain, and that the different contradicting realities could coexist ${ }^{27}$. However, the measuring devices used in this experiment were not conscious, since the "observers", used were recording and measuring devices. Nevertheless, the scientists, who carried out this experiment, argue that these devices can carry out measurements, and define an observer, as a physical system capable of extracting physical information, via interaction, and then storing it on a physical memory ${ }^{28}$.

Therefore, although this experiment shows that the contradicting realities can coexist, the experiment is inconclusive in relation to whether this occurs with conscious observers.

\footnotetext{
27 Daily Nous. (2019). Philosophers On a Physics Experiment that "Suggests There's No Such Thing As Objective Reality" - Daily Nous. [online] Available at: http://dailynous.com/2019/03/21/philosophers-physics-experiment-suggests-theres-no-thing-objectivereality/ [Accessed 22 Aug. 2019].

28 Daily Nous. (2019). Philosophers On a Physics Experiment that "Suggests There's No Such Thing As Objective Reality" - Daily Nous. [online] Available at: http://dailynous.com/2019/03/21/philosophers-physics-experiment-suggests-theres-no-thing-objectivereality/ [Accessed 22 Aug. 2019].
} 
Hence what is needed to adequately test whether the paradox, defined in Wigner's friend can remain, is to create a variant of the experiment being mentioned here, in which the measuring devices are conscious. Therefore, this aspect of premise 3 is empirically testable, since it is entirely possible to design, a variation of this experiment but with conscious beings, which would ultimately put to the test whether, the paradox can just exist as separate realities.

Additionally, one could also, formulate the objection that a paradox can just stay as a philosophical, contradiction, meaning that, the conundrum could just prevail in one reality, rather than, existing in separate realities. Essentially, a case could be made that a God is not needed to collapse the paradox, because due to some philosophically absurd reason, the paradox just remains as a paradox (in this reality), and thus doesn't need collapsing. This notion, which although is not necessarily scientifically testable, is logically absurd. It can therefore be dismissed. Hence, although there, are some objections, which can be made that are not empirically testable (such as the latter), ultimately premise 3 , is scientifically confirmable, since many of these objection are just philosophical nonsense, and can be easily dismissed. This is primarily because such objections are not exclusive to the ideas outlined in this paper, and could be levelled against any scientific theory.

Premise, 3 is therefore scientifically verifiable, because the idea that the paradox can just coexist in separate realities, could be confirmed or not confirmed by an experiment which meet the requirements for an experiment to be scientific, in the introduction. Furthermore, the other part of the premise, which is that it could exist as a philosophical contradiction, is just philosophical speculation, and would be no different, to the speculation that could be used to discredit highly established scientific theories. Therefore, these philosophical objections would not hinder the scientific testability of this premise. Moreover, this premise would contribute, to the scientific proof of God's existence, because if this assumption were empirically verified, it would remove a major philosophical and scientific possibility, which is that the paradox can remain, and in turn that a higher cause, such as an ultimate consciousness is needed to resolve the paradox.

\section{4) Justify that the wave function of the initial particle in Wigner's friend (and the first observer) has undoubtedly collapsed:}

The last area that needs to be scientifically confirmable is that wave function collapse actually occurs. This needs to be validated, in the sense that the wave function of the first observer (Wigner's friend), and the wave function of the particle, which is in a

superposition state of having decayed and not decayed, has collapsed. This is imperative since, by knowing that the collapse of the wave function has occurred (which means that there is no paradox present), we are able to deduce from the previous premises that this collapse occurred due to an ultimate form of consciousness otherwise known as God.

One way in, which this could be substantiated, is to determine, from the perspective of observer 2 whether, it has collapsed, by checking for circumstantial evidence that there is a superposition, such as interference. Firstly this would involve, the initial observer confirming, that it has collapsed after him consciously viewing the state of the particle. However the primary observer mustn't specify the particular state it has collapsed to. Then, in order to test whether the particle's wave function has unambiguously collapsed the second viewer without knowing the result or having the potential to know the result, would have to check for whether there is any circumstantial evidence that indicates that there is or isn't a superposition. Such as whether the decay states interfere. Moreover, the optimal way to test this, would be to check for these circumstantial properties (e.g. interference), throughout the entire duration of the experiment. This way we will know for certain that it has collapsed because, throughout the entire, time span of the experiment there was no indication that the particle was in superposition. This can be further, applied to the superposition of observer 1 as well. Therefore, this premise is scientifically 
testable, since it meets the requirements for it to be, which are outlined in the previous section.

Additionally, a check for interference would involve, the second observer somehow conducting the experiment, from his location to test whether, there is interference. If this premise, were verified, it would complete the endeavour of scientifically proving God's existence, since it would have confirmed, that there isn't a paradox, and as was explained in previous premises, if there is no paradox, this can only be due to an ultimate observer. Therefore, with the scientific verification of the antecedent premises, which when added together allow us to empirically arrive at the conclusion that the only way in which the paradox, in question cannot be present, is by virtue of invocation of an ultimate observer (God), and with the empirical verification that in reality there actually is no paradox present with the confirmation of this premise, then the conclusion has to be arrived at, that scientifically God exists. Since it has determined that something which could only happen as a consequence of an ultimate observer(God), viewing it has indeed happened. Namely that there is no paradox as defined in the Wigner's friend thought experiment. 


\section{Conclusion}

In the introduction, of this paper, it was specified that this article, would attempt, to outline a possible way in which the existence of God could be proven scientifically. God, was defined as the creator and ruler of the universe. This target has been some what achieved. By, showing that the required premises, for Wigner's friend when added together undoubtedly lead to the existence of an ultimate observer (in the introduction), which is outside of the natural world (and above other metaphysical minds), and that these premises are scientifically testable, this paper has shown that it is possible to prove the existence of a type of ultimate form of consciousness. In summary, it is possible to scientifically establish, that the only way in which the paradox, that is described in Wigner's friend could not be present, is by the invocation of a supreme form of consciousness or God. Then by empirically verifying that a paradox is not there by confirming that the wave function of the particle in Wigner's friend has collapsed, a scientific proof of God's existence would have been reached. This is because, the empirical confirmation that the only way in which a paradox could not be present is by invoking an ultimate observer, would imply that when it is scientifically discovered that no paradox is there, that an ultimate observer (or God), is real since his existence is necessary for there not to be a paradox. Hence it is possible to scientifically test the hypothesis of God.

However, this paper, hasn't been able to demonstrate that the nature of this supreme form of consciousness could be tested scientifically, in regard to whether it is the creator and ruler of the universe. Therefore, this paper has to arrive at the conclusion that the adaptation of Wigner's friend can only scientifically prove that there is an ultimate form of consciousness that it above space and time, and also above all metaphysical minds. It cannot be scientifically verified, using this adaptation of Wigner's friend whether this ultimate form of consciousness, is God as is defined in the introduction. It therefore seems unlikely that another experiment, adopting similar principles used in this adaptation of Wigner's friend, could potentially scientifically prove the existence of God (defined as the creator and ruler of the universe).

Furthermore it, must also be said that based on this line of thinking, it seems entirely plausible, that other 'philosophical', claims can actually be proven scientifically. These claims, might include moral statements, or any philosophical issue, ranging from miracles, to religious language. If these issues, are broken down into verifiable premises, as was done in this paper, then potentially these assertions could be confirmed or not confirmed, scientifically. 


\section{References:}

Bradford, A. (2019). What Is Science?. [online] livescience.com. Available at: https://www.livescience.com/20896-science-scientific-method.html [Accessed 17 Aug. 2019].

chlosshauer, M., Kofler, J. and Zeilinger, A. (2013). A snapshot of foundational attitudes toward quantum mechanics. Studies in History and Philosophy of Science Part B: Studies in History and Philosophy of Modern Physics, 44(3)

Daily Nous. (2019). Philosophers On a Physics Experiment that "Suggests There's No Such Thing As Objective Reality" - Daily Nous. [online] Available at: http://dailynous.com/2019/03/21/philosophersphysics-experiment-suggests-theres-no-thing-objective-reality/ [Accessed 22 Aug. 2019].

Dirac, P. (1947). The Principles of Quantum Mechanics (2nd edition). Clarendon Press.

En.wikipedia.org. (2019). Existence of God. [online] Available at: https://en.wikipedia.org/wiki/Existence_of_God\#cite_ref-46 [Accessed 22 Aug. 2019].

Informationphilosopher.com. (2019). [online] Available at: http://www.informationphilosopher.com/solutions/scientists/wigner/Wigner_Remarks.pdf [Accessed 22 Aug. 2019].

Lexico Dictionaries | English. (2019). God | Definition of God in English by Lexico Dictionaries. [online] Available at: https://www.lexico.com/en/definition/god [Accessed 22 Aug. 2019].

Li, T. and Yin, Z. (2016). Quantum superposition, entanglement, and state teleportation of a microorganism on an electromechanical oscillator. Science Bulletin, 61(2)

Philosophynow.org. (2019). The Integrated Information Theory of Consciousness | Issue $121 \mid$ Philosophy Now. [online] Available at: https://philosophynow.org/issues/121/The_Integrated_Information_Theory_of_Consciousness [Accessed 18 Aug. 2019].

Physics World. (2019). Could 'Schrödinger's bacterium' be placed in a quantum superposition? Physics World. [online] Available at: https://physicsworld.com/a/could-schrdingers-bacterium-beplaced-in-a-quantum-superposition/ . [Accessed 22 Aug. 2019].

Plato.stanford.edu. (2001). Bohmian Mechanics (Stanford Encyclopedia of Philosophy). [online] Available at: plato.stanford.edu/entries/qm-bohm/ [Accessed 16 Aug. 2019].

Plato.stanford.edu. (2002). Many-Worlds Interpretation of Quantum Mechanics(Stanford Encyclopedia of Philosophy). [online] Available at: plato.stanford.edu/entries/qm-manyworlds/ [Accessed 16 Aug. 2019].

Plato.stanford.edu. (2019). Ontological Arguments (Stanford Encyclopedia of Philosophy). [online] Available at: https://plato.stanford.edu/entries/ontological-arguments/ [Accessed 16 Aug. 2019]. 
Plato.stanford.edu. (2019). Scientific Method (Stanford Encyclopedia of Philosophy). [online] Available at: https://plato.stanford.edu/entries/scientific-method/\#LogMetCriRes [Accessed 16 Aug. 2019].

Schr dinger, E. (1935). Die gegenw rtige Situation in der Quantenmechanik. Die

Naturwissenschaften, 23(48)

The UCSB Current. (2019). A Tiny Machine. [online] Available at: https://www.news.ucsb.edu/2016/017349/tiny-machine [Accessed 22 Aug. 2019].

YouTube. (2019). Wigner's Friend Paradox: Is Observation Inherently Flawed?. [online] Available at: https://www.youtube.com/watch?v=5AodzEpvzZw [Accessed 22 Aug. 2019]. 